\title{
Note on Ethnic Names and Languages
}

With minor exceptions, Ugandan and South African languages belong to two broad families: the Bantu family and the northwestern branch of the Indo-European family of languages. Thus, all of the languages within these families have basic grammar in common.

Southern Uganda represents the northernmost reach of the Bantu languages, while South Africa represents their southernmost reach. Bantu languages use prefixes to designate specific meanings for general, or root, terms. Thus Ganda is the root name of the largest ethnic group and kingdom in southern Uganda, and prefixes denote its various aspects. For example, Luganda is the Ganda language, Buganda is the Ganda kingdom or territory, and Baganda is the plural for Ganda people, or the Ganda nation or tribe.

Similarly, in South Africa, Zulu is a root name from which other terms are derived. For example, IsiZulu is the Zulu language, and AmaZulu is the Zulu people or nation (especially the followers of the Zulu king, particularly those living in the northern part of the province of KwaZulu-Natal). These prefixes vary somewhat from language to language. Thus, SeSotho is the name of the Sotho language, where the $s e$ - prefix is cognate to the isi-prefix in IsiZulu.

Besides Luganda, English is the other dominant language in Uganda. In both South Africa and Uganda, English is the primary language of education, especially at the secondary school and university levels, and the primary language of commerce. In everyday practice, however, 
South African language use involves considerable mixing of at least four languages: IsiZulu, English, Afrikaans, and SeSotho. Although there are officially eleven languages in South Africa, all others are either closely related to these four or are mutually intelligible. Most South Africans and Ugandans use multiple languages in daily life. 